\title{
FKBP3 mediates oxaliplatin resistance in colorectal cancer cells by regulating HDAC2 expression
}

\author{
JIAFENG TONG $^{1 *}$, YING SHEN $^{2 *}, \mathrm{XI} \mathrm{CHEN}^{3}, \mathrm{RENJIE} \mathrm{WANG}^{4}, \mathrm{YE} \mathrm{HU}^{2}$, \\ XU ZHANG ${ }^{1}$, ZHENGHUA ZHANG ${ }^{5}$ and LI HAN $^{1}$

\begin{abstract}
${ }^{1}$ Department of Traditional Chinese Medicine, Huadong Hospital, Fudan University, Shanghai 200040;
${ }^{2}$ Department of Gastroenterology, Shanghai Municipal Hospital of Traditional Chinese Medicine Affiliated to

Shanghai 200040; ${ }^{4}$ Department of Colorectal Surgery, Fudan University Shanghai Cancer Center,
\end{abstract} \\ Shanghai TCM University, Shanghai 200071; ${ }^{3}$ Department of Oncology, Huadong Hospital, Fudan University, \\ Shanghai 200030; ${ }^{5}$ Department of Oncology, Jing'an District Central Hospital of Shanghai (Huashan Hospital, \\ Fudan University, Jing'an Branch), Shanghai 200040, P.R. China
}

Received September 4, 2018; Accepted June 26, 2019

DOI: $10.3892 /$ or.2019.7259

\begin{abstract}
Colorectal cancer (CRC), a commonly occurring malignant tumor in the gastrointestinal tract, is the third leading cause of cancer-related deaths worldwide. FK506-binding proteins (FKBPs) comprise an immunophilin family that are involved in the carcinogenesis, progression and chemoresistance of cancers, including CRC. FKBP3 (also known as FKBP25) is a nuclear protein that is a member of the FKBP family and is correlated with the activity of histone deacetylase 2 (HDAC2). However, the role of FKBP3 and HDAC2 in oxaliplatin resistance in CRC and the potential molecular mechanisms are still poorly understood. In the present study, the expression of FKBP3, HDAC2 and related-genes was detected by real-time PCR and western blot analysis. Furthermore, cell apoptosis was detected by flow cytometry (FCM). We found high expression of FKBP3 and HDAC2 in CRC tissues. In vitro, primary CRC cells with high expression of FKBP3 and HDAC2 were insensitive to oxaliplatin. Downregulation of FKBP3 significantly increased the sensitivity of primary CRC cells to oxaliplatin, reduced expression of HDAC2, permeability glycoprotein
\end{abstract}

Correspondence to: $\mathrm{Dr} \mathrm{Li}$ Han, Department of Traditional Chinese Medicine, Huadong Hospital, Fudan University, 221 Yan'an West Road, Jing'an, Shanghai 200040, P.R. China

E-mail: tcmhan2018@sina.com

Dr Zhenghua Zhang, Department of Oncology, Jing'an District Central Hospital of Shanghai (Huashan Hospital, Fudan University, Jing'an Branch), 259 Xikang Road, Shanghai 200040, P.R. China E-mail: 18916731691@189.com

*Contributed equally

Key words: FKBP3, HDAC2, oxaliplatin-resistance, colorectal cancer, apoptosis, PTEN/AKT pathway
(P-gp) and phosphorylated AKT (p-AKT), and increased expression of phosphatase and tensin homolog (PTEN) and cleaved caspase-3. Accordingly, upregulation of FKBP3 had the opposite effect. Furthermore, downregulation of HDAC2 significantly counteracted FKBP3-induced oxaliplatin resistance in CRC cells. Our data revealed that oxaliplatin resistance in CRC cells is positively associated with FKBP3 and HDAC2 expression, and FKBP3 downregulation could attenuate oxaliplatin resistance in $\mathrm{CRC}$ cells by reducing HDAC2 expression and possibly through regulation of the PTEN/AKT pathway.

\section{Introduction}

Colorectal cancer (CRC), a commonly occurring malignant tumor in the gastrointestinal tract, is the third leading cause of cancer-related deaths worldwide, causing approximately 600,000 deaths each year $(1,2)$. Each year, more than one million new CRC patients are diagnosed worldwide (3), and in China, especially in underdeveloped areas, the incidence of CRC is increasing rapidly (4). Currently, oxaliplatin-based chemotherapy is the primary strategy for CRC treatment; however, patients ultimately relapse due to drug resistance (5). Oxaliplatin is reported to be the first platinum compound to be effective for the treatment of CRC (6), through inhibition of tumor cell growth and G2-phase cell cycle arrest (7). However, resistance to oxaliplatin poses a huge challenge to $\mathrm{CRC}$ treatment, and its underlying mechanism remains unclear.

Phosphatase and tensin homolog deleted on chromosome 10 (PTEN) is a lipid phosphatase that frequently serves as a tumor suppressor in multiple human cancers (8). It has been reported that PTEN is of great significance in the progression of CRC $(9,10)$. PTEN can antagonize the action of the AKT pathway, and studies have implicated the PTEN/AKT pathway to be associated with drug resistance in several types of cancer $(11,12)$. For example, microRNA-21 was found to overcome sorafenib-resistance of liver cancer cells through the PTEN/AKT pathway (11). In addition, overexpression of 
microRNA-22 was able to reverse paclitaxel-resistance of CRC cells by activating PTEN (12).

FK506-binding proteins (FKBPs), an immunophilin family, bind immunosuppressive drugs and are involved in various processes, including cancer progression and chemoresistance (13). It has been shown that FKBP51 can inhibit the cell proliferation in CRC (14). Additionally, the tumorigenesis and chemoresistance of cancers can be negatively regulated by USP49 through FKBP51/AKT signaling $(15,16)$. FKBP3 (also known as FKBP25), a nuclear member of the FKBP family, has been shown to transcriptionally regulate the expression of p53 and p21 (17). Previously, it was also reported to be associated with the activity of histone deacetylases $1 / 2$ (HDAC1/2) (18). HDACs are an ancient superfamily of enzymes containing multiple members including HDAC1 and HDAC2, and are involved in the development and progression of human cancers (19). Overexpression of HDACs has been observed in a variety of human cancers such as CRC (20), and downregulation of HDAC2 was found to inhibit the cell growth of liver cancer cells by the PTEN/PI3K/AKT pathway (21). In addition, a study revealed that FKBP3 promotes lung cancer cell proliferation through the regulation of Sp1/HDAC2/p27 signaling (22). However, the role of FKBP3 and HDAC2 in oxaliplatin resistance in CRC and its potential molecular mechanisms are still poorly understood.

Here we showed that FKBP3 and HDAC2 are highly expressed in CRC tissues. Compared to CRC cells with low FKBP3 expression, CRC cells with high FKBP3 expression were more insensitive to oxaliplatin treatment, concurrent with decreased cleaved caspase-3 and increased B-cell lymphoma-2 (Bcl-2) expression. Downregulation of FKBP3 significantly increased apoptosis in oxaliplatin-resistant CRC cells, reduced the expression levels of HDAC2, permeability glycoprotein (P-gp) and phosphorylated AKT (p-AKT), and increased expression of PTEN and cleaved caspase-3. Furthermore, downregulation of HDAC2 reversed FKBP3-induced oxaliplatin resistance in CRC cells. Our data demonstrated that FKBP3 and HDAC2 expression increased oxaliplatin resistance in $\mathrm{CRC}$ cells, and that the downregulation of FKPB3 re-sensitized cells to oxaliplatin by reducing HDAC2 expression, and possibly through the regulation of the PTEN/AKT pathway.

\section{Materials and methods}

CRC tissues and adjacent normal tissues. Samples from a total of $58 \mathrm{CRC}$ patients (age range, 40-70 years; 30 males and 28 females) were collected from June 2017 to June 2018). All patients enrolled in this study were treated at Shanghai Municipal Hospital of Traditional Chinese Medicine Affiliated to Shanghai TCM University (Shanghai, China. Tumor and adjacent normal tissues were collected from these patients after the receipt of written informed consent. Tissues were frozen and stored in liquid nitrogen immediately before use. After total RNA extraction, the expression of FKBP3 and HDAC2 in tissues was detected. All experiments conducted in this study were approved by the Ethics Committee of Shanghai Municipal Hospital of Traditional Chinese Medicine Affiliated to Shanghai TCM University (Shanghai, China).
Cell culture. After obtaining written informed consent, primary cells were isolated from 12 CRC patients treated at Shanghai Municipal Hospital of Traditional Chinese Medicine Affiliated to Shanghai TCM University (Shanghai, China). The cells were cultured in a $5 \% \mathrm{CO}_{2}$ incubator (Thermo Fisher Scientific, Inc., Waltham, MA, USA) at $37^{\circ} \mathrm{C}$ with HyClone ${ }^{\mathrm{TM}}$ high glucose DMEM medium (cat. no. SH30243.01; Thermo Fisher Scientific, Inc.) supplemented with Gibco ${ }^{\mathrm{TM}} 10 \%$ fetal bovine serum (FBS; cat. no. 16000-044; Thermo Fisher Scientific, Inc.) and 1\% antibiotic (penicillin and streptomycin; cat. no. P1400-100; Beijing Solarbio Science \& Technology Co., Ltd., Beijing, China). The medium was refreshed every two days during culture.

Construction of lentiviral plasmids. shRNA plasmids were constructed by synthesizing and inserting sequences targeting FKBP3 (5'-GAGGTTCAATGTTGGATAT-3') and HDAC2 (5'-CCCAUAACUUGCUGUUAAA-3') (22) into AgelI/EcoRI restriction sites of a pLKO.1-puro vector. The coding DNA sequence (CDS) region of FKBP3 (NM_002013.3) with the addition of EcoRI and BamHI restriction sites was synthesized, by Genewiz Co. (Shanghai, China), and inserted into the EcoRI/BamHI sites of a pLVX-Puro vector (Clontech). After confirmation by DNA sequencing (Shanghai Majorbio Pharmaceutical Technology Co., Ltd., Shanghai, China), pLKO.1-Puro-shFKBP3, pLKO.1-Puro-shHDAC2 or pLVX-Puro-FKBP3 was co-transfected into 293T cells with viral packaging plasmids psPAX2 and pMD2G (Addgene, Inc., Cambridge, MA, USA) using Lipofectamine 2000 (Invitrogen; Thermo Fisher Scientific, Inc.). pLKO.1-Puro and pLVX-Puro were used as negative control vectors. The virus particles in the supernatant were collected by ultracentrifugation $48 \mathrm{~h}$ after transfection.

Experimental grouping. Primary CRC cells with high expression of FKBP3 were divided into four groups and infected with FKBP3 overexpression (oeFKBP3)/vector and FKBP3 interference (shFKBP3)/negative control (shNC) lentiviruses. Forty-eight hours after infection, the efficiency of oeFKBP3 and shFKBP3, as well as the expression of HDAC2 were evaluated by real-time PCR and western blot analysis.

To further investigate the role of FKBP3 and HDAC2 in oxaliplatin resistance in $\mathrm{CRC}$, the primary $\mathrm{CRC}$ cells with high expression of FKBP3 were treated with shNC+vector $+40 \mu \mathrm{g} / \mathrm{ml}$ oxaliplatin (O124003; Aladdin), shFKBP3+40 $\mu \mathrm{g} / \mathrm{ml}$ oxaliplatin, or oeFKBP3 $+40 \mu \mathrm{g} / \mathrm{ml}$ oxaliplatin. Primary cells were also treated with shNC+vector+40 $\mu \mathrm{g} / \mathrm{ml}$ oxaliplatin, oeFKBP3 $+40 \mu \mathrm{g} / \mathrm{ml}$ oxaliplatin, shHDAC $2+40 \mu \mathrm{g} / \mathrm{ml}$ oxaliplatin, or oeFKBP $3+\operatorname{shHDAC} 2+40 \mu \mathrm{g} / \mathrm{ml}$ oxaliplatin. Twenty-four hours after oxaliplatin treatment, cell apoptosis and the expression of related-proteins were quantified, and the 24-h treatment of oxaliplatin had a significant decrease in apoptosis in CRC primary cells, and thus this time point was selected for further experiments.

Real-time polymerase chain reaction ( $q P C R)$ assay. Total RNA from primary CRC cells or treated cells was extracted using TRIzol reagent (cat. no. 1596-026; Invitrogen; Thermo Fisher Scientific, Inc.). After quantification, the integrity of extracted RNA was confirmed by electrophoresis using a $1 \%$ 
gel. Subsequently, $1 \mu \mathrm{g}$ of RNA was reverse transcribed into cDNA using a Reverse Transcription Kit (\#K1622; Fermentas; Thermo Fisher Scientific, Inc.). Using the cDNA as a template, the qPCR reactions were conducted on a Real-time PCR system (cat. no. ABI-7300; Applied Biosystems; Thermo Fisher Scientific, Inc.) with a SYBR-Green PCR kit (\#K0223; Thermo Fisher Scientific, Inc.). The mRNA expression levels of FKBP3 and HDAC2, normalized to GAPDH, were calculated by the $2^{-\triangle \triangle C q}$ method (23). The primers used are listed as follows: FKBP3 forward, 5'-ACCCAAAGAAACCAAGTC-3' and reverse, 5'-ATACCAGCAGTGAACAAC-3'; HDAC2 forward, 5'-GCTGGGATTACAGGTGTGAG-3' and reverse, 5'-AGGCTGAGGTGGGAGAATAC-3'; GAPDH forward, 5'-AATCCCATCACCATCTTC-3' and reverse, 5'-AGGCTG TTGTCATACTTC-3'. The qPCR program was as follows: $95^{\circ} \mathrm{C}$ for $10 \mathrm{~min}\left(95^{\circ} \mathrm{C}\right.$ for $15 \mathrm{sec} ; 60^{\circ} \mathrm{C}$ for $\left.45 \mathrm{sec}\right) \mathrm{x} 40 ; 95^{\circ} \mathrm{C}$ for $15 \mathrm{sec} ; 60^{\circ} \mathrm{C}$ for $1 \mathrm{~min} ; 95^{\circ} \mathrm{C}$ for $15 \mathrm{sec} ; 60^{\circ} \mathrm{C}$ for $15 \mathrm{sec}(24)$.

Western blot analysis. Western blot analysis was performed as previously described to determine the levels of related-proteins in CRC cells (25). Total proteins from primary CRC cells or treated cells were extracted using RIPA buffer containing protease and phosphatase inhibitors (cat. no. R0010, Solarbio, Beijing, China). Following quantification by a BCA Kit (cat. no. PICPI23223; Thermo Fisher Scientific, Inc.), $25 \mu \mathrm{g}$ of protein were separated on 10 or $8 \%$ SDS-polyacrylamide gels. Subsequently, proteins were transferred to polyvinylidene fluoride (PVDF) membranes (cat. no. HATF00010; EMD Millipore, Billerica, MA, USA) using a semi-dry transfer and blocked in 5\% skim milk (cat. no. BYL40422BD; Biosciences, Franklin Lakes, NJ, USA) at room temperature for $1 \mathrm{~h}$. The membranes were incubated with primary antibodies against FKBP3 (dilution 1:2,000; cat. no. Ab16654; Abcam, Cambridge, UK), HDAC2 (dilution 1:2,000; cat. no. Ab32117, Abcam), PTEN (dilution 1:500; cat. no. Ab31392; Abcam), P-gp (dilution 1:200; cat. no. 103477; Abcam), AKT [dilution 1:1,000; cat. no. 4685; Cell Signaling Technology (CST), Inc., Danvers, MA, USA], p-AKT (dilution 1:2,000; cat. no. 4060; CST), cleaved caspase-3 (dilution 1:1,000; cat. no. Ab13847; Abcam), and GAPDH (dilution 1:2,000; cat. no. 5174; CST) overnight at $4^{\circ} \mathrm{C}$. The next day, the membranes were washed 5-6 times and incubated with HRP-labeled goat anti-rabbit (cat. no. A0208) and goat anti-mouse (catalog no. A0216) secondary antibodies (dilution 1:1,000; Beyotime Institute of Biotechnology, Haimen, China) at room temperature for $2 \mathrm{~h}$. After 5-6 washes, the blots were developed with a chemiluminescent reagent (cat. no. WBKLS0100; EMD Millipore) for $5 \mathrm{~min}$ prior to exposure on an ECL imaging system (Tanon-5200; Tanon Science and Technology Co., Ltd., Shanghai, China). GAPDH served as an internal control; the expression of proteins was analyzed and calculated by ImageJ software (version $1.47 \mathrm{v}$; National Institutes of Health, Bethesda, MD, USA).

Cell apoptosis assay. Apoptosis in the treated primary CRC cells was evaluated by flow cytometric (FCM) analysis. After treatment, primary CRC cells were collected to conduct a Annexin V-fluorescein isothiocyanate (FITC)/propidium iodide (PI) double stain (C1063; Beyotime Institute of Biotechnology) according to the manufacturer's protocol. Approximately $5 \times 10^{5}-1 \times 10^{6}$ cells were counted and then
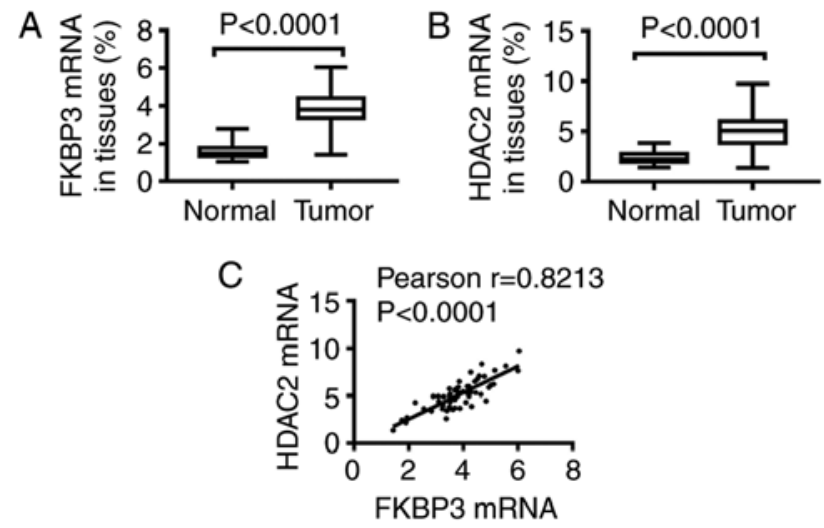

Figure 1. mRNA expression of FKBP3 and HDAC2 is significantly increased in tumors of CRC patients. Tumor and adjacent normal tissues from CRC patients were collected. (A and B) Expression of FKBP3 and HDAC2 at the mRNA level was detected by qPCR $(\mathrm{P}<0.0001)$. (C) The correlation between FKBP3 and HDAC2 was determined by Pearson analysis. The data are shown as mean $\pm \mathrm{SD}$ of three experiments. CRC, colorectal cancer; FKBP3, FK506-binding protein 3; HDAC2, histone deacetylase 2.

resuspended in $195 \mu 1$ Annexin V-FITC binding buffer. Subsequently, the cells were incubated with $5 \mu 1$ Annexin V-FITC for $15 \mathrm{~min}$ at $4^{\circ} \mathrm{C}$ in the dark prior to incubation in $5 \mu \mathrm{l} \mathrm{PI}$ for 5 min. Cells without Annexin V-FITC and PI staining were used as a negative control. Using BD Accuri ${ }^{\mathrm{TM}}$ C6 Software (version 1.0.264.21; BD Biosciences, USA), the percentage of apoptosis in primary CRC cells was analyzed and evaluated on a flow cytometer.

Statistical analysis. GraphPad Prism 7.0 software (GraphPad Software, Inc., La Jolla, CA, USA) was applied in this study to analyze the statistical significance. The significance between two groups was analyzed by two-tailed Student's t-test, while one-way analysis of variance (ANOVA) with a post-test of Tukey's multiple comparison were used for three or more groups. Pearson's correlation analysis was also applied for the correlation between the two groups. Based on three independent experiments, data are expressed as mean \pm SD and P-values $<0.05$ were defined to be statistically significant.

\section{Results}

Expression of FKBP3 and HDAC2 is significantly increased in tumors of CRC patients. After RNA extraction of tissues, qPCR assay was performed to detect FKBP3 and HDAC2 expression. As demonstrated in Fig. 1, we found that, compared to normal tissues, the expression of FKBP3 (Fig. 1A) and HDAC2 (Fig. 1B) at the mRNA level in tumors was significantly increased. Importantly, Pearson analysis showed that the expression of HDAC2 was positively correlated to FKBP3 expression (Fig. 1C). This suggests that FKBP3 and HDAC2 are critically important in CRC progression.

High expression of FKBP3 increases resistance to oxaliplatin in primary CRC cells. Previous studies have shown that oxaliplatin resistance in cancers displays different behaviors, including inhibition of apoptosis (26). Here, we evaluated apoptosis levels to assess Ooxaliplatin resistance in CRC cells. We isolated primary cells from 12 CRC tissues and examined 
A

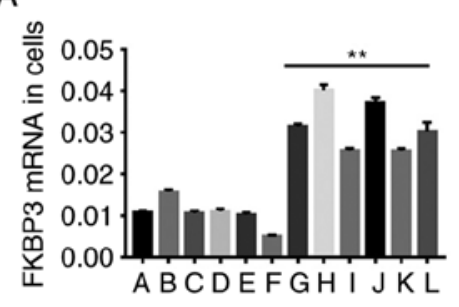

D
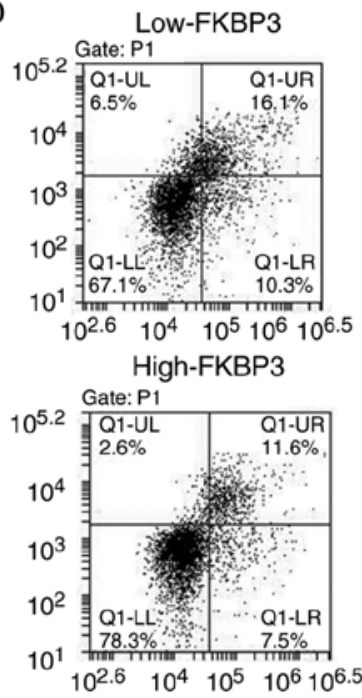

B

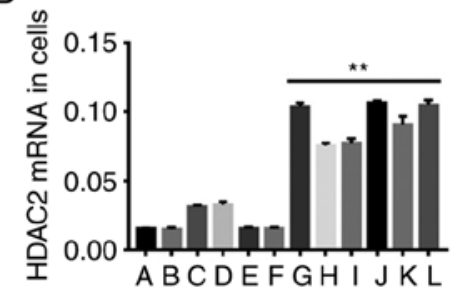

E
C A B C D E F G H I J K L

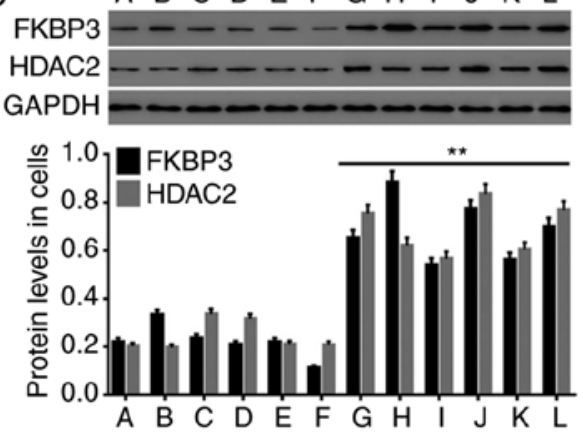

A B C D E F G H I J K L

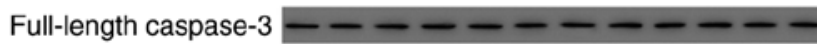
Cleaved caspase-3

$\mathrm{BCl}-2$ $\mathrm{GAPDH}---\infty-\infty-\infty$ Full-length caspase-3 Cleaved caspase- 3 ***

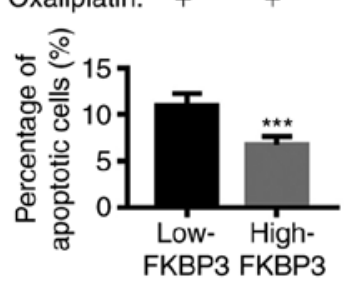

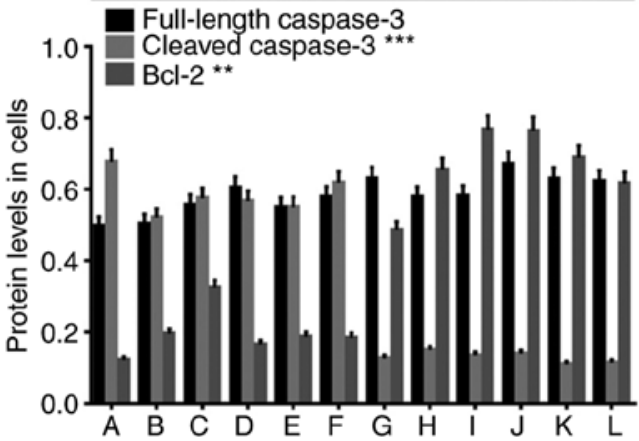

Figure 2. High expression of FKBP3 increases oxaliplatin resistance in primary CRC cells. Expression analysis of FKBP3 and HDAC2 was conducted in 58 primary CRC cell lines. Six highly-expressing (G, H, I, J, K, L) and 6 lowly-expressing (A, B, C, D, E, F) cell lines were selected for further study. (A and B) The mRNA expression of FKBP3 and HDAC2 was detected by qPCR. (C) The protein levels of FKBP3 and HDAC2 were detected by western blot analysis. (D) After treatment with $40 \mu \mathrm{g} / \mathrm{ml}$ of oxaliplatin, the CRC cells were incubated with Annexin V-FITC/PI, and the percentage of apoptotic CRC cells was calculated by FCM analysis. The lower right (LR) quadrant represents early apoptotic cells positively stained with Annexin V, while the upper right (UR) quadrant represents late apoptotic or necrotic cells doubly stained with Annexin V and PI. The lower left (LL) quadrant shows living cells. (E) The protein expression of caspase-3, cleaved caspase- 3 and Bcl-2 in CRC cells was detected. All data are presented as mean \pm SD of three experiments. ${ }^{* *} \mathrm{P}<0.01$ and ${ }^{* * *} \mathrm{P}<0.001$ compared to Low-FKBP3 (A-F: Low-FKBP3, G-L: High-FKBP3). CRC, colorectal cancer; FKBP3, FK506-binding protein 3; HDAC2, histone deacetylase 2; Bcl-2, B-cell lymphoma-2.

the expression levels of FKBP3 and HDAC2. We found that both FKBP3 (Fig. 2A and C) and HDAC2 (Fig. 2B and C) were highly expressed in 6 primary CRC cell lines $(\mathrm{G}, \mathrm{H}$, I, J, K, L), and relatively lowly expressed in another 6 cell lines (A, B, C, D, E, F). Subsequently, the cells were treated with oxaliplatin at $40 \mu \mathrm{g} / \mathrm{ml}$ for $24 \mathrm{~h}$. The results in Fig. 2D showed that, compared to CRC cells with low FKBP3 expression (A-F), the percentage of apoptotic cells in CRC cells with high FKBP3 expression (G-L) was significantly decreased. Concurrently, the expression of cleaved caspase-3, a cysteine protease that has been reported to be an important regulator for programmed cell death or apoptosis, was significantly decreased in CRC cells with high FKBP3 expression, and $\mathrm{Bcl}-2$, an antiapoptotic protein, was increased, while caspase-3 was unchanged (Fig. 2E) (27,28). This suggests that oxaliplatin resistance in CRC cells may be positively associated with FKBP3 and HDAC2 expression.

Downregulation and upregulation of FKBP3 in primary $C R C$ cells by lentiviral infection. Primary CRC cells were infected with shFKBP3/shNC or oeFKBP3/vector lentiviruses. The results in Fig. 3 show that the expression of FKBP3 (Fig. 3A and C) at the mRNA and protein levels in primary CRC cells was markedly downregulated by shFKBP3 and upregulated by oeFKBP3. In addition, the expression of HDAC2 in primary CRC cells was positively regulated by FKBP3 expression (Fig. 3B and C). This indicates that HDAC2 is a possible downstream target of FKBP3, which can be regulated by FKBP3. shFKBP3 and oeFKBP3 lentiviruses were used for the following experiments.

Downregulation of FKBP3 decreases oxaliplatin resistance in CRC cells and regulates the PTEN/AKT pathway. The underlying mechanisms of FKBP3 in regulating oxaliplatin resistance in CRC cells were investigated. We downregulated or upregulated the expression of FKBP3 in a primary CRC cell line and found that downregulation of FKBP3 significantly promoted apoptosis in oxaliplatin-resistance CRC cells, whereas FKBP3 upregulation had the opposite effect (Fig. 4A). Several related-proteins were also assessed. PTEN, as a tumor suppressor, is a potent inhibitor of the AKT pathway (29). It has been reported that PTEN regulates cellular processes such as apoptosis in cancers through the AKT pathway (30-32). $\mathrm{P}$-gp, encoded by multidrug resistance-associated protein (MRP), is a major indicator of drug resistance, and caspase-3 is one of the major apoptosis-executing enzymes. As shown 

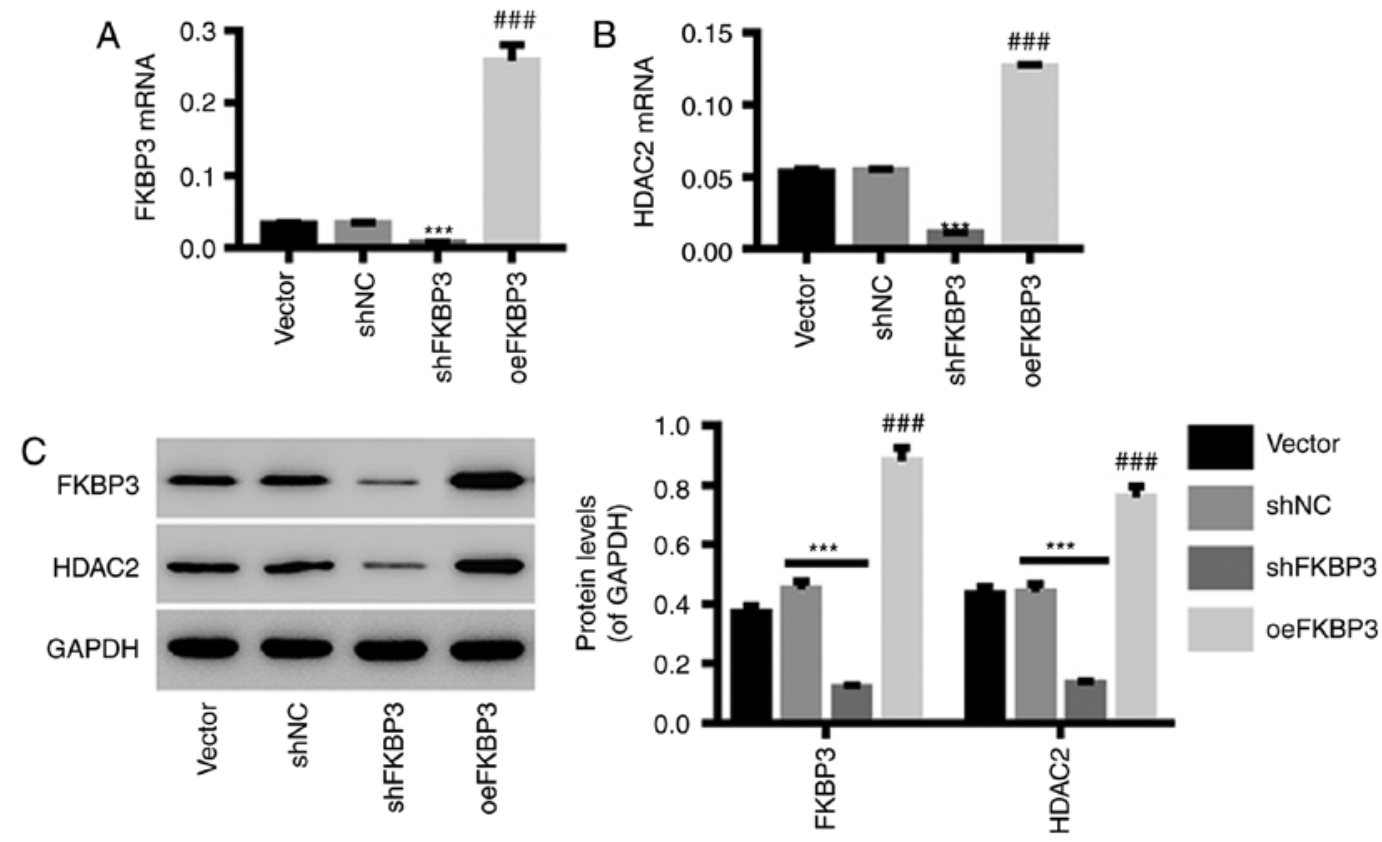

Figure 3. Downregulation and upregulation of FKBP3 in primary CRC cells by lentiviral infection. Primary CRC cells were infected with shFKBP3/shNC or oeFKBP3/vector lentiviruses in vitro. (A and B) The mRNA expression of FFKBP3 and HDAC2 was detected by qPCR. (C) The protein levels of FKBP3 and HDAC2 were detected by western blot analysis. The results are presented as mean \pm SD of three experiments. ${ }^{* * * *} \mathrm{P}<0.001$ compared to shNC; ${ }^{\# \# *} \mathrm{P}<0.001$ compared to vector. CRC, colorectal cancer; FKBP3, FK506-binding protein 3; HDAC2, histone deacetylase 2.

in Fig. 4B, downregulation of FKBP3 significantly decreased HDAC2 and P-gp expression, and increased cleaved caspase-3 expression, while full-length caspase- 3 was unchanged, and FKBP3 upregulation had the opposite effect. Furthermore, downregulation of FKBP3 decreased AKT phosphorylation and increased PTEN expression in oxaliplatin-resistant CRC cells, while the expression of AKT was unchanged (Fig. 4C). This demonstrated that downregulation of FKBP3 can increase the sensitivity of CRC cells to oxaliplatin and FKBP3 possibly regulates the PTEN/AKT pathway in oxaliplatin-resistant CRC cells.

FKBP3-induced resistance to oxaliplatin in CRC cells is mediated by HDAC2. Next, we knocked down the expression HDAC2 in CRC cells (Fig. 5A) and found that downregulation of HDAC2 also significantly increased apoptosis in oxaliplatin-resistant primary CRC cells (Fig. 5B), accompanied by decreased expression of P-gp and p-AKT, and increased expression of PTEN and cleaved caspase-3 (Fig. 5C and D), and unchanged full-length caspase-3, which was similar to FKBP3 downregulation. In addition, FKBP3-induced inhibition of apoptosis in oxaliplatin-treated CRC cells was significantly counteracted by HDAC 2 downregulation. These results further revealed that downregulation of FKBP3 can attenuate the resistance of $\mathrm{CRC}$ cells to oxaliplatin by reducing HDAC2 expression.

\section{Discussion}

Studies have demonstrated that several members of the FK506-binding proteins (FKBPs) such as FKBP38, FKBP52 and FKBP65 potentially play an important role in the tumor progression and its acquisition of chemoresistance $(33,34)$. For example, FKBP38, a noncanonical member of the
FKBP family, contributes to tumorigenesis and chemoresistance by interacting with B-cell lymphoma-2 (Bcl-2) (34). In the present study, elevated expression of FKBP3 and histone deacetylases 2 (HDAC2) was observed in colorectal cancer (CRC) tissues. Primary CRC cells with high FKBP3 and HDAC2 expression were insensitive to oxaliplatin, suggesting that oxaliplatin resistance in CRC cells may be positively associated with FKBP3 and HDAC2 expression. Furthermore, we showed that downregulation of FKBP3 or HDAC2 significantly increased the sensitivity of CRC cells to oxaliplatin by promoting apoptosis, which is in agreement with previous reports $(15,16,33,34)$, suggesting that FKBP3 and HDAC2 may be involved in chemotherapy resistance in primary CRC cells.

We also explored the underlying mechanisms by which FKBP3 downregulation attenuates the resistance of CRC cells to oxaliplatin. It has previously been reported that FKBP3 is correlated with the activity of HDAC2 (18), which modulates histone acetylation, thus regulating the expression of apoptosis-related genes $(35,36)$. Consistent with these reports, our study found that downregulation of FKBP3 in CRC cells markedly decreased HDAC2 and P-glycoprotein (P-gp) expression, and increased expression of pro-apoptotic molecule, cleaved caspase-3, whereas FKBP3 upregulation had an opposite effect. This suggests that HDAC2 is a possible downstream effector of FKBP3 and may be involved in the carcinogenicity and drug-resistant capabilities of FKBP3 in CRC. In addition, downregulation of HDAC2 significantly increased the sensitivity of primary CRC cells to oxaliplatin by promoting apoptosis, accompanied by decreased expression of P-gp and p-AKT, and increased expression of phosphatase and tensin homolog deleted on chromosome 10 (PTEN) and cleaved caspase-3. Furthermore, HDAC2 downregulation significantly attenuated the effects of FKBP3 upregulation, providing 
A
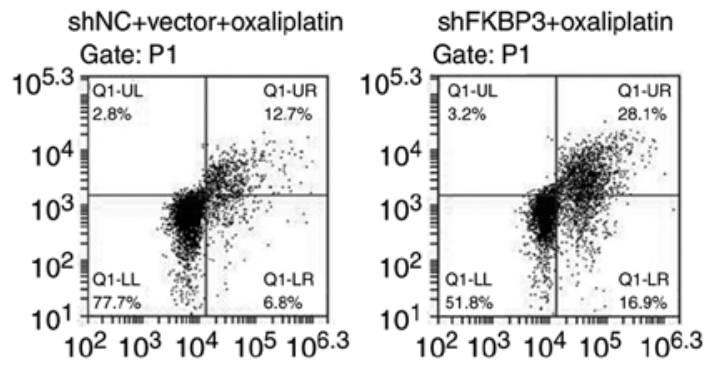

oeFKBP3+oxaliplatin
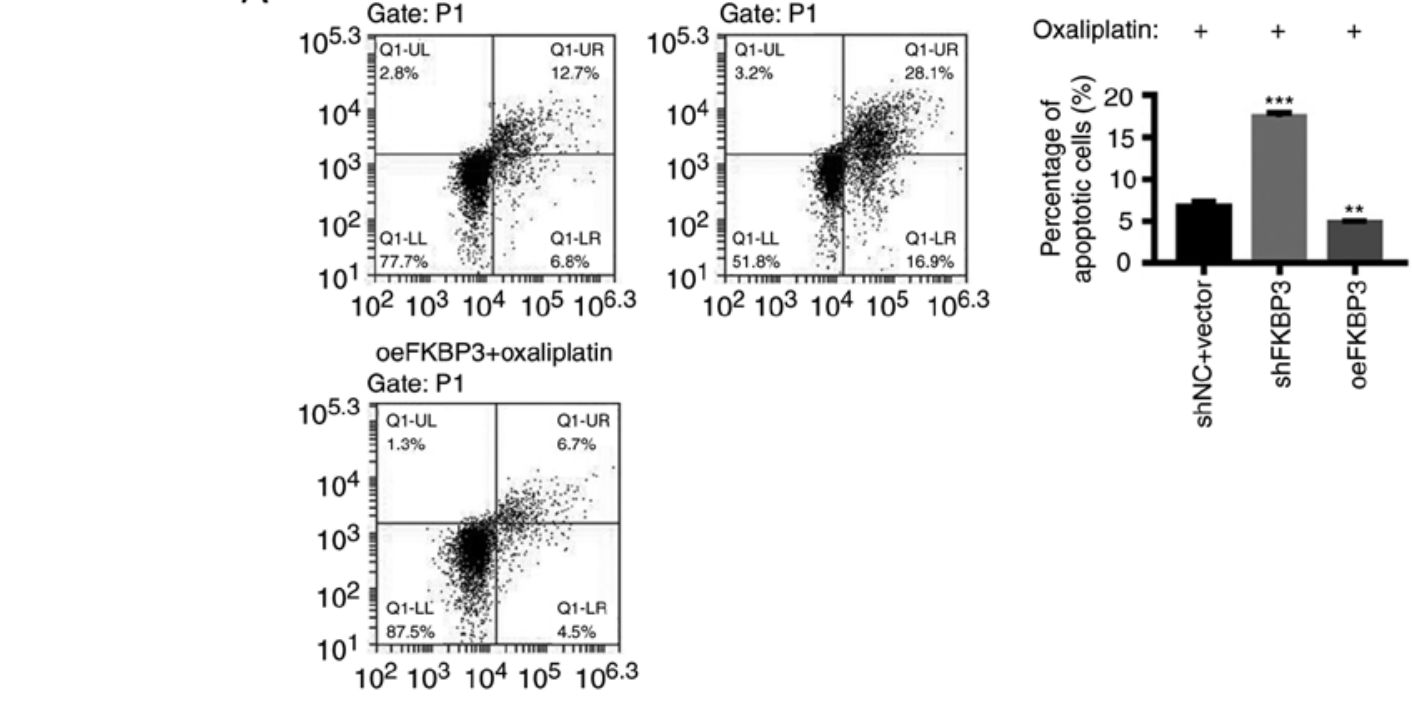

C

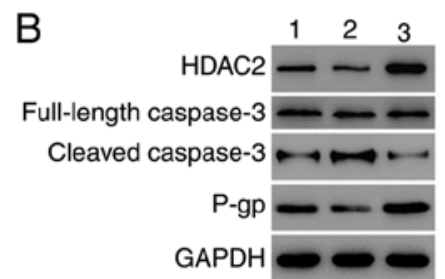

1: shNC+vector+oxaliplatin 2: shFKBP3+oxaliplatin 3: oeFKBP3+oxaliplatin

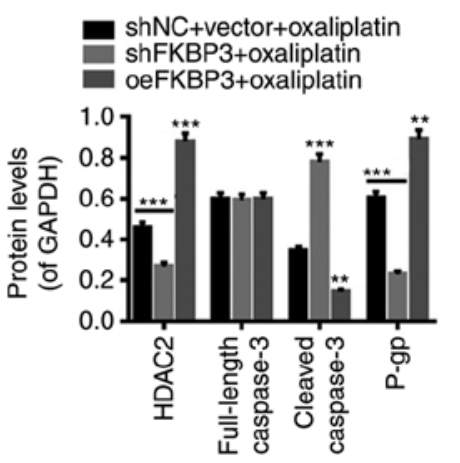

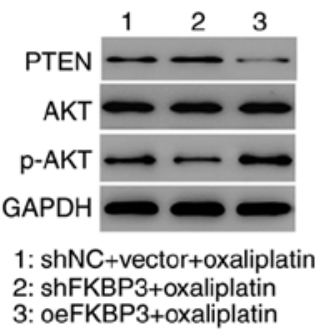

2: shFKBP3+oxaliplatin

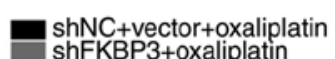
shFKBP3+oxaliplatin

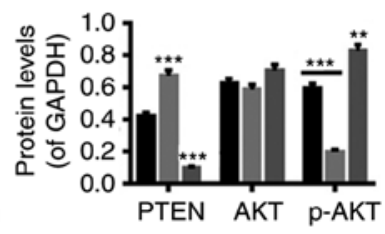
PTEN AKT p-AKT

Figure 4. Downregulation of FKBP3 decreases oxaliplatin resistance in CRC cells and regulates the PTEN/AKT pathway. CRC cells were treated with shNC+vector $+40 \mu \mathrm{g} / \mathrm{ml}$ oxaliplatin, shFKBP3+40 $\mu \mathrm{g} / \mathrm{ml}$ oxaliplatin, or oeFKBP3 $+40 \mu \mathrm{g} / \mathrm{ml}$ oxaliplatin for $48 \mathrm{~h}$. (A) After incubation with Annexin V-FITC/PI, the percentage of apoptotic cells was evaluated by FCM analysis. (B and C) The levels of HDAC2, PTEN, P-gp, full-length caspase-3, cleaved caspase-3, p-AKT and AKT were detected by western blot analysis. All data are expressed as mean $\pm \mathrm{SD}$ of three experiments. ${ }^{* *} \mathrm{P}<0.01$, ${ }^{* * *} \mathrm{P}<0.001$ compared to shNC+vector. CRC, colorectal cancer; FKBP3, FK506-binding protein 3; HDAC2, histone deacetylase 2; PTEN, phosphatase and tensin homolog deleted on chromosome 10; P-gp, P-glycoprotein.

further evidence that FKBP3 regulates the resistance of CRC cells to oxaliplatin, possibly through modulation of HDAC2 expression. It has been reported that FKBP3 functionally associates with histone deacetylases, such as HDAC1 and HDAC2, and through the recruitment of HDAC1 or HDAC2, FKBP3 could free up acetylated lysine residues in mouse double minute 2 homolog (MDM2), making them available for auto-ubiquitylation $(18,37)$. Finally, we found that downregulation of FKBP3 significantly decreased AKT phosphorylation and increased PTEN expression in oxaliplatin-resistant CRC cells. A recent study has shown that the PI3K/AKT pathway is associated with chemotherapy resistance, mainly through evasion of cellular apoptosis (38). PTEN can negatively regulate the $\mathrm{PI} 3 \mathrm{~K} / \mathrm{AKT}$ pathway by dephosphorylating PIP3 to PIP2, preventing all downstream signaling events that are regulated by AKT $(39,40)$. It is well-known that the PI3K/AKT pathway is involved in the regulation of cell proliferation and survival, and constitutively active AKT can protect cells from apoptosis by suppressing caspase-3, as well as decreasing the sensitivity of tumor cells to pro-apoptotic agents $(38,41)$. AKT signaling was also discovered to be appreciably activated in tumors and functions in drug resistance (42). Therefore, we inferred that FKBP3 could regulate the PTEN/AKT pathway, and that the PTEN/AKT pathway may be involved in FKBP3 regulation of chemoresistance in CRC cells; however further experiments are needed to definitively address this conclusion. For example, after inhibition or activation of PTEN/AKT, FCM should be used to analyze the apoptosis in FKBP3-overexpressing or -silenced CRC cells, as well as to detect related proteins by western blotting. There are also limitations in the present study; for example, the lack of time points of oxaliplatin treatment, reference of shFKBP3 interference sequence, as well as the lack of experiments for supporting our hypothesis. If possible in the future, several types of shFKBP3 targeting different sequences will be tested to select shRNAs with optimal interference effect, and more time points of oxaliplatin treatment should be tested in the future to make our research more credible. Moreover, an inhibitor of PETN/AKT can be applied to further study the mechanisms of FKBP3, HDAC2 and the PTEN/AKT pathway in CRC.

In conclusion, the present study demonstrated the critically important role of FKBP3 and HDAC2 in the resistance of CRC cells to oxaliplatin. Downregulation of FKBP3 can attenuate the resistance of CRC cells to oxaliplatin by reducing HDAC2 expression. Moreover, FKBP3 can possibly regulate 
A

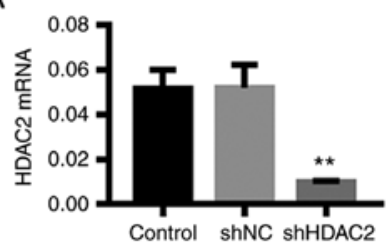

HDAC2

GAPDH

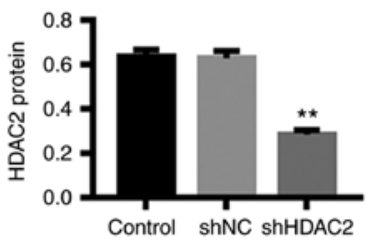

B

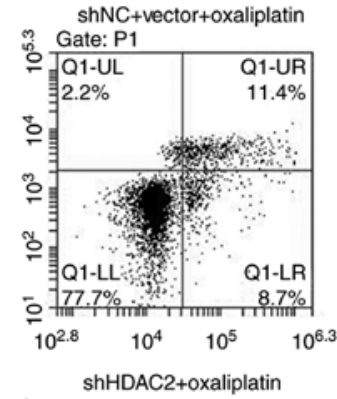

\%) Gate: P1

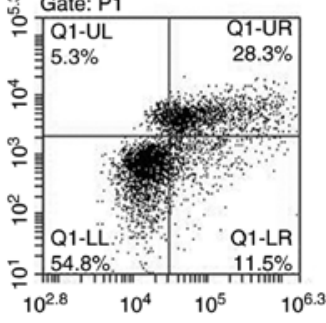

C

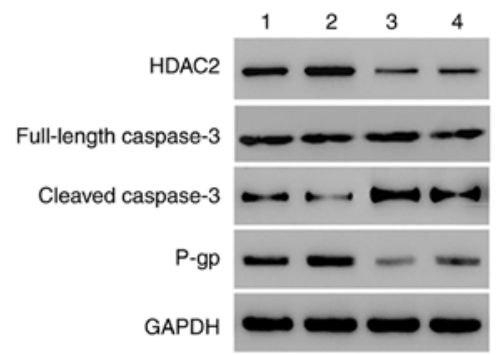

1: shNC+vector+oxaliplatin

2: oeFKBP3+oxaliplatin

3: shHDAC2+oxaliplatin

4: oeFKBP3+shHDAC2+oxaliplatin

D

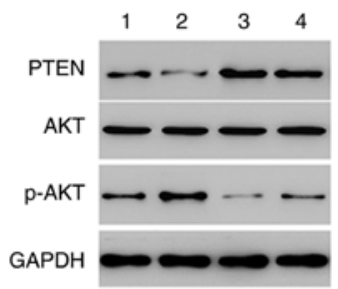

1: shNC+vector+oxaliplatin

2: oeFKBP3+oxaliplatin

3: shHDAC2+oxaliplatin

4: oeFKBP3+shHDAC2+oxaliplatin
oeFKBP3+oxaliplatin

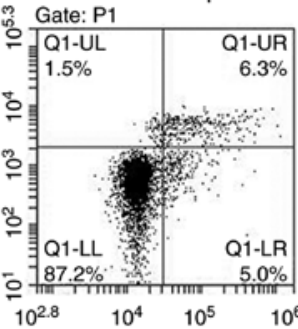

oeFKBP3+shHDAC2+oxaliplatin \%. Gate: P1
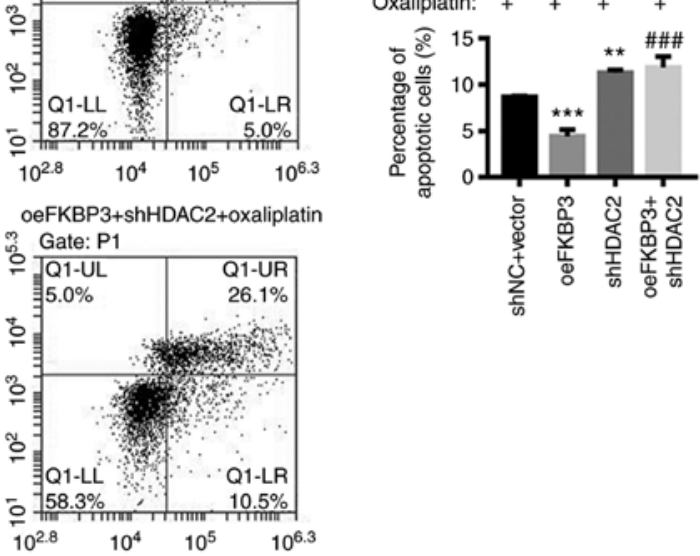

Figure 5. FKBP3-induced resistance to oxaliplatin in CRC cells is mediated by HDAC2. CRC cells were treated with shNC+vector+40 $\mu \mathrm{g} / \mathrm{ml}$ oxaliplatin, oeFKBP3 $+40 \mu \mathrm{g} / \mathrm{ml}$ oxaliplatin, shHDAC $2+40 \mu \mathrm{g} / \mathrm{ml}$ oxaliplatin, or oeFKBP3+shHDAC2+40 $\mu \mathrm{g} / \mathrm{ml}$ oxaliplatin for $48 \mathrm{~h}$. (A) The expression of HDAC2 mRNA and protein was detected. (B) After incubation with Annexin V-FITC/PI, the percentage of apoptotic cells was evaluated by FCM analysis. (C and D) The levels of HDAC2, PTEN, P-gp, full-length caspase-3, cleaved caspase-3, p-AKT and AKT were detected by western blot analysis. All data are shown as mean $\pm \mathrm{SD}$ of three experiments. ${ }^{* *} \mathrm{P}<0.01,{ }^{* * *} \mathrm{P}<0.001$ compared to shNC or shNC+vector; ${ }^{\# \#} \mathrm{P}<0.001$ compared to oeFKBP3. CRC, colorectal cancer; FKBP3, FK506-binding protein 3; HDAC2, histone deacetylase 2; PTEN, phosphatase and tensin homolog deleted on chromosome 10; P-gp, P-glycoprotein. 
the PTEN/AKT pathway, and the PTEN/AKT pathway may be involved in FKBP3 regulation of chemoresistance in CRC cells. Importantly, our study suggests the possible clinical value and potential prognosis of FKBP3 and HDAC2 in chemotherapeutic resistance of $\mathrm{CRC}$, which may contribute to CRC treatment.

\section{Acknowledgements}

Not applicable.

\section{Funding}

This study was funded by the Clinical Study on Chang Ji Tai Combined with Auricular Acupuncture in Treating Postoperative Cancer-Related Fatigue of Postoperative Colorectal Cancer, Special Item of Shanghai Integrative Medicine [ZY (2018-2020)-FWTX-3016].

\section{Availability of data and materials}

All data generated or analyzed during this study are included in this published article.

\section{Authors' contributions}

$\mathrm{ZZ}$ and $\mathrm{LH}$ conceived and designed the study. JT, YS, XC, $\mathrm{RW}, \mathrm{YH}$ and $\mathrm{XZ}$ performed the experiments, and collected and analyzed the data. $\mathrm{ZZ}$ and LH wrote the manuscript. All authors read and approved the final manuscript and agree to be accountable for all aspects of the research in ensuring that the accuracy or integrity of any part of the work are appropriately investigated and resolved.

\section{Ethics approval and consent to participate}

All experiments conducted in this study were approved by the Ethics Committee of Shanghai Municipal Hospital of Traditional Chinese Medicine Affiliated to Shanghai TCM University (Shanghai, China), and written informed consent was obtained.

\section{Patient consent for publication}

Not applicable.

\section{Competing interests}

The authors declare that they have no competing interests.

\section{References}

1. Ung L, Lam KY, Morris DL and Chua TC: Tissue-based biomarkers predicting outcomes in metastatic colorectal cancer: a review. Clin Transl Oncol 16: 425-435, 2014.

2. Sostres C, Gargallo CJ and Lanas A: Aspirin, cyclooxygenase inhibition and colorectal cancer. World J Gastrointest Pharmacol Ther 5: 40-49, 2014

3. Siegel RL, Miller KD and Jemal A: Cancer statistics, 2016. CA Cancer J Clin 66: 7-30, 2016.

4. Chen W, Zheng R, Baade PD, Zhang S, Zeng H, Bray F, Jemal A $\mathrm{Yu}$ XQ and He J: Cancer statistics in China, 2015. CA Cancer J Clin 66: 115-132, 2016
5. Overman MJ, Morris V, Moinova H, Manyam G, Ensor J, Lee MS, Eng C, Kee B, Fogelman D, Shroff RT, et al: Phase I/II study of azacitidine and capecitabine/oxaliplatin (CAPOX) in refractory CIMP-high metastatic colorectal cancer: Evaluation of circulating methylated vimentin. Oncotarget 7: 67495-67506, 2016.

6. Meyerhardt JA and Mayer RJ: Systemic therapy for colorectal cancer. N Engl J Med 352: 476-487, 2005.

7. Kelland L: The resurgence of platinum-based cancer chemotherapy. Nat Rev Cancer 7: 573-584, 2007.

8. Koul D, Shen R, Bergh S, Sheng X, Shishodia S, Lafortune TA, $\mathrm{Lu} \mathrm{Y,} \mathrm{de} \mathrm{Groot} \mathrm{JF,} \mathrm{Mills} \mathrm{GB} \mathrm{and} \mathrm{Yung} \mathrm{WK:} \mathrm{Inhibition} \mathrm{of} \mathrm{Akt}$ survival pathway by a small-molecule inhibitor in human glioblastoma. Mol Cancer Ther 5: 637-644, 2006.

9. Xiong $\mathrm{F}$ and Chen J: Effect of miR-3651 on proliferation, apoptosis and expression of PTEN in colon cancer cells. Chin Clin Oncol 22: 865-868, 2017.

10. Liang YF, Jian-Bo R, Wang LM, Chen C, Kang DP, Lin BH, Chai XX, Zeng JC and Pathology DO: Expression and clinical significance of PTEN in colon cancer. China Tropical Med 15: 966-969, 2015.

11. He C, Dong X, Zhai B, Jiang X, Dong D, Li B, Jiang H, Xu S and Sun X: MiR-21 mediates sorafenib resistance of hepatocellular carcinoma cells by inhibiting autophagy via the PTEN/Akt pathway. Oncotarget 6: 28867-28881, 2015.

12. Li J, Zhang Y, Zhao J, Kong F and Chen Y: Overexpression of miR-22 reverses paclitaxel-induced chemoresistance through activation of PTEN signaling in p53-mutated colon cancer cells. Mol Cell Biochem 357: 31-38, 2011.

13. Solassol J, Mange A and Maudelonde T: FKBP family proteins as promising new biomarkers for cancer. Curr Opin Pharmacol 11: 320-325, 2011.

14. Mukaide H, Adachi Y, Taketani S, Iwasaki M, Koi-Kekiriyama N, Shigematsu A, Shi M, Yanai S, Yoshioka K, Kamiyama Y and Ikehara S: FKBP51 expressed by both normal epithelial cells and adenocarcinoma of colon suppresses proliferation of colorectal adenocarcinoma. Cancer Invest 26: 385-390, 2008.

15. Li L, Lou $\mathrm{Z}$ and Wang L: The role of FKBP5 in cancer aetiology and chemoresistance. Br J Cancer 104: 19-23, 2011.

16. Luo K, Li Y, Yin Y, Lei L, Wu C, Chen Y, Nowsheen S, Qi H, Zhang L, Lou Z and Yuan J: USP49 negatively regulates tumorigenesis and chemoresistance through FKBP51-AKT signaling. EMBO J 36: 1434-1446, 2017.

17. Ochocka AM, Kampanis P, Nicol S, Allende-Vega N, Cox M, Marcar L, Milne D, Fuller-Pace F and Meek D: FKBP25, a novel regulator of the p53 pathway, induces the degradation of MDM2 and activation of p53. FEBS Lett 583: 621-626, 2009.

18. Yang WM, Yao YL and Seto E: The FK506-binding protein 25 functionally associates with histone deacetylases and with transcription factor YY1. EMBO J 20: 4814-4825, 2001.

19. Oiso H, Furukawa N, Suefuji M, Shimoda S, Ito A, Furumai R, Nakagawa J, Yoshida M, Nishino N and Araki E: The role of class I histone deacetylase (HDAC) on gluconeogenesis in liver. Biochem Biophys Res Commun 404: 166-172, 2011.

20. Jeong JB and Lee SH: Protocatechualdehyde possesses anti-cancer activity through downregulating cyclin D1 and HDAC2 in human colorectal cancer cells. Biochem Biophys Res Commun 430: 381-386, 2013.

21. Zhang H, Zhao B, Huang C, Meng XM, Bian EB and Li J: Melittin restores PTEN expression by down-regulating HDAC2 in human hepatocelluar carcinoma HepG2 cells. PLoS One 9: e95520, 2014.

22. Zhu W, Li Z, Xiong L, Yu X, Chen X and Lin Q: FKBP3 promotes proliferation of non-small cell lung cancer cells through regulating Sp1/HDAC2/p27. Theranostics 7: 3078-3089, 2017.

23. Livak KJ and Schmittgen TD: Analysis of relative gene expression data using real-time quantitative PCR and the 2(-Delta Delta C(T)) method. Methods 25: 402-408, 2001

24. Hong JY, Kang B, Kim A, Hwang S, Ahn J, Lee S, Kim J, Park JH and Cheon DS: Development of a highly sensitive real-time one step RT-PCR combined complementary locked primer technology and conjugated minor groove binder probe. Virol J 8: 330, 2011.

25. Xiong H, Hong J, Du W, Lin YW, Ren LL, Wang YC, Su WY, Wang JL, Cui Y, Wang ZH and Fang JY: Roles of STAT3 and ZEB1 proteins in E-cadherin down-regulation and human colorectal cancer epithelial-mesenchymal transition. J Biol Chem 287: 5819-5832, 2012.

26. Virag P, Brie I, Fischer-Fodor E, Perde-Schrepler M, Tatomir C, Balacescu O, Irimie A and Postescu ID: Assessment of cytotoxicity, apoptosis and DNA damages in Colo320 colorectal cancer cells selected for oxaliplatin resistance. Cell Biochem Funct 29: 351-355 2011. 
27. Cohen GM: Caspases: The executioners of apoptosis. Biochem J 326: 1-16, 1997.

28. Gao G and Dou QP: G(1) phase-dependent expression of bcl-2 mRNA and protein correlates with chemoresistance of human cancer cells. Mol Pharmacol 58: 1001-1010, 2000.

29. Lee MS, Jeong MH, Lee HW, Han HJ, Ko A, Hewitt SM, Kim JH, Chun KH, Chung JY, Lee C, et al: PI3K/AKT activation induces PTEN ubiquitination and destabilization accelerating tumourigenesis. Nat Commun 6: 7769, 2015.

30. Salmena L, Carracedo A and Pandolfi PP: Tenets of PTEN tumor suppression. Cell 133: 403-414, 2008.

31. Davies MA, Lu Y, Sano T, Fang X, Tang P, Lapushin R, Koul D, Bookstein R, Stokoe D, Yung WK, et al: Adenoviral transgene expression of MMAC/PTEN in human glioma cells inhibits akt activation and induces anoikis. Cancer Res 58: 5285-5290, 1998.

32. Mehrian-Shai R, Chen CD, Shi T, Horvath S, Nelson SF, Reichardt JK and Sawyers CL: Insulin growth factor-binding protein 2 is a candidate biomarker for PTEN status and PI3K/Akt pathway activation in glioblastoma and prostate cancer. Proc Natl Acad Sci USA 104: 5563-5568, 2007.

33. Choi BH and Yoon HS: FKBP38-Bcl-2 interaction: A novel link to chemoresistance. Curr Opin Pharmacol 11: 354-359, 2011.

34. Ma D, Bai X, Zou H, Lai Y and Jiang Y: Rheb GTPase controls apoptosis by regulating interaction of FKBP38 with Bcl-2 and Bcl-XL. J Biol Chem 285: 8621-8627, 2010.

35. Krämer $\mathrm{OH}$, Knauer SK, Zimmermann D, Stauber RH and Heinzel T: Histone deacetylase inhibitors and hydroxyurea modulate the cell cycle and cooperatively induce apoptosis. Oncogene 27: 732-740, 2008.

36. Jung KH, Noh JH, Kim JK, Eun JW, Bae HJ, Xie HJ, Chang YG, Kim MG, Park H, Lee JY and Nam SW: HDAC2 overexpression confers oncogenic potential to human lung cancer cells by deregulating expression of apoptosis and cell cycle proteins. J Cell Biochem 113: 2167-2177, 2012.
37. Wang X, Taplick J, Geva N and Oren M: Inhibition of p53 degradation by Mdm2 acetylation. FEBS Lett 561: 195-201, 2004.

38. Fresno Vara JA, Casado E, de Castro J, Cejas P, Belda-Iniesta C and González-Barón M: PI3K/Akt signalling pathway and cancer. Cancer Treat Rev 30: 193-204, 2004.

39. Gan YH and Zhang S: PTEN/AKT pathway involved in histone deacetylases inhibitor induced cell growth inhibition and apoptosis of oral squamous cell carcinoma cells. Oral Oncol 45: e150-e154, 2009.

40. Song MS, Salmena L and Pandolfi PP: The functions and regulation of the PTEN tumour suppressor. Nat Rev Mol Cell Biol 13: 283-296, 2012.

41. van Echten-Deckert G, Zschoche A, Bär T, Schmidt RR, Raths A, Heinemann $\mathrm{T}$ and Sandhoff $\mathrm{K}$ : cis-4-Methylsphingosine decreases sphingolipid biosynthesis by specifically interfering with serine palmitoyltransferase activity in primary cultured neurons. J Biol Chem 272: 15825-15833, 1997.

42. Nemoto S, Nakamura M, Osawa Y, Kono S, Itoh Y, Okano Y, Murate T, Hara A, Ueda H, Nozawa Y and Banno Y: Sphingosine kinase isoforms regulate oxaliplatin sensitivity of human colon cancer cells through ceramide accumulation and Akt activation. J Biol Chem 284: 10422-10432, 2009.

This work is licensed under a Creative Commons Attribution-NonCommercial-NoDerivatives 4.0 International (CC BY-NC-ND 4.0) License. 